\title{
EDITORIAL
}

\section{LA ESCRITURA Y EL ARTE DE LA DISCIPLINA}

Llegamos a la edición 50 de la revista Estudios de Literatura Colombiana, siempre puntuales, con nuestras dos ediciones por año, en enero y julio, respectivamente. En resumidas cuentas, la revista celebra 25 años en los que ha sido testigo del devenir de la literatura y la cultura del país. Testigos de esta historia nuestra, cruenta pero también vital. Y habría mucho en qué pensar, pero aquí nos vamos a decantar por la disciplina, a propósito de este cuarto de siglo, pues esta es crucial para los editores, y ha sido una de las claves para seguir llegando siempre con la misma puntualidad a nuestros lectores, desde aquella primera edición publicada en 1997. Al fin de cuentas, la disciplina es una de las claves para quien escribe, para quien investiga, pero sobre todo es esencial para un buen ejercicio de la ciudadanía, algo que parece precario en nuestro país, a juzgar por la imagen caótica que tiende a imponerse en nuestro entorno, en todos los niveles, desde los asuntos macro de nuestra política y nuestra economía, por ejemplo, hasta los detalles más mínimos de nuestra cotidianidad, como el simple hecho de pasarse un semáforo en rojo, o pegarse de la bocina inmediatamente aparece la luz verde. Esos simples actos pueden ser más dicientes que cualquier teoría.

Pero hablemos de la disciplina en la escritura. José Luis Díaz-Granados (2017) se refiere a la metódica costumbre que le facilitó a Gabriel García Márquez convertirse en uno de los más célebres escritores de la segunda mitad del siglo xx:

Cuando Gabo decidió trasladarse a Barcelona, le puso orden definitivo a sus costumbres creadoras y desafiando el asedio constante de periodistas e intrusos, estableció entre nueve de la mañana y dos de la tarde, una severa autodisciplina con la que logró "las virtudes de concisión y transparencia de estilo" de El otoño del patriarca.

En adelante, se las arregló para no alterar jamás su disciplina férrea de escritor (Díaz-Granados, 2017).

Una afirmación confirmada por Jaime García Márquez cuando, al referirse a la clave para el éxito de su hermano, afirma que "Gabito es hechura de su disciplina", y agrega que él era "Disciplina, disciplina pura" (Yajure, 2013). Y esto no debe sorprendernos, pues más allá de la genialidad que pueda tener un escritor, el único camino para hacer una obra tan prolífica y tan consistente - aunque tengamos que reconocer que su poder narrativo menguó en sus últimas producciones— es su capacidad de mantenerse enfocado en su objetivo. 
La periodista y escritora Alma Guillermoprieto, quien lo conoció de cerca desde que él la llamó para que participara en la construcción de la Fundación Nuevo Periodismo Iberoamericano, con motivo del adiós al autor de El coronel no tiene quien le escriba evocó el rigor que él ponía en su escritura:

En sus mañanas García Márquez armaba y rearmaba la ingeniería de un párrafo, corrigiendo y reforzando cada punto de apoyo hasta dejarlo prácticamente antisísmico. Estudió siempre a los autores que admiraba, de la misma forma que examinan los atletas los videos de los demás Medallas de Oro, no para copiar sino para entender. Pero antes de cualquier lectura, y mucho antes que la primera frase que tecleó algún día, tuvo entre sus muy principales herramientas a su prodigiosa memoria, que lo surtía no solo de recuerdos, sino de palabras. Tenía miles en su haber - sueltas, guardadas en arcones o hiladas en secuencia como si fuera en collares - y las recordaba todas (Guillermoprieto, 2014).

Y uno de los autores preferidos de García Márquez, Ernest Hemingway, también se destaca por su rigor ejemplar. Mario Escobar Velásquez en su taller de escritura alguna vez nos presentó como una gran lección de disciplina una anécdota al respecto, aunque no recuerdo cuáles títulos mencionó: cuando el autor de El viejo y el mar llegó al final de una de sus obras, como todavía no había cumplido con la meta diaria, con su lápiz trazó una línea horizontal en mitad de la página y comenzó el siguiente relato. Otra anécdota que refleja su entrega a la escritura son las 47 versiones que escribió para el final de Adiós a las armas, porque "Buscaba las palabras adecuadas" (Hemingway, 1996, p. 89), como contó él mismo en una entrevista para The Paris Review, aunque hay que aclarar que en dicha entrevista él mencionó 39 finales, pero después se pudo confirmar que en realidad fueron ocho más, pues "las 47 versiones se encuentran archivadas en la Colección Ernest Hemingway de la Biblioteca y Museo John Fitzgerald Kennedy de Boston" (Saiz, 2012). Él insistía en el ejercicio de revisar y pulir, como un tallador: "La relectura me pone en el sitio en el que la escritura tiene que seguir, sabiendo que hasta allí todo está tan bien como es posible. Siempre queda resto en alguna parte" (Hemingway, 1996, p. 89).

Y a propósito de Mario Escobar, una de las cosas en las que más nos insistía en su taller era en la disciplina con la lectura y la escritura, y uno de los mandamientos para sus discípulos era que tuviéramos siempre una agenda a la mano, fuéramos a donde fuéramos, para que pudiéramos escribir cuando nos asaltaran las ideas. Y él mismo aplicaba con fe ese mandamiento. Siempre andaba con su agenda, y no era raro encontrarlo en una mesa de la Universidad de Antioquia haciendo sus apuntes. Un fruto de esa práctica es el Diario de un escritor, publicado por la Editorial Univer- 
sidad de Antioquia con algunos extractos de aquellas agendas. Y precisamente uno de esos apuntes constituye esta honda confesión, que nos ilustra muy bien sobre su rigor:

Por ahora mi disciplina descansa de mi novela chilapa. Como el descanso no la amaña, pronto irá a fustigarme porque inicie a otra. No veo de qué construirla. Las últimas tres, inéditas, tuvieron el mismo acoso que presiento. El cerebro acabó respondiendo. En cada vez pensé que no habría material. Acaso así suceda ahora. Y, a veces, unas ganas enormes de gritarle a la disciplina: "no me joda más. ¿Para qué otra novela?”.

¿Para qué otra novela? Pudiera responderme que para sentirme vivo en tanto la escribo (Escobar Velásquez, pp. 336-337).

Por su parte William Faulkner, cuando en 1956 Jean Stein, en una entrevista para The Paris Review, le preguntó si había alguna fórmula posible para convertirse en un buen novelista, le respondió:

Noventa y nueve por ciento de talento... Noventa y nueve por ciento de disciplina... Noventa y nueve por ciento de trabajo. Nunca hay que estar satisfecho con lo que hace. Nunca es tan bueno como podría serlo. Siempre hay que soñar y apuntar más alto de lo que es posible hacer. No hay que preocuparse simplemente por ser mejor que los contemporáneos o que los predecesores. Hay que tratar de ser mejor que uno mismo. Un artista es una criatura impulsada por los demonios. Nunca se sabe por qué lo eligieron a él y suele estar demasiado ocupado como para preguntárselo. Es completamente amoral en el sentido de que puede llegar a robar, a pedir prestado o a mendigar ante cualquiera para poder hacer su obra (Faulkner, 1996, p.170).

En Escribir, un desgarrador testimonio, Marguerite Duras ratifica la importancia de ejercer la escritura todos los días, con dedicación. En este ensayo también pone de relieve que escribir es un acto en la soledad, la cual se vuelve necesaria para el escritor: "La soledad de la escritura es una soledad sin la que el escribir no se produce, o se fragmenta exangüe de buscar qué seguir escribiendo. Se desangra, el autor deja de reconocerlo" (Duras, 1994, p. 16).

Y nuestro invitado a la sesión de entrevista en la presente edición, Darío Ruiz Gómez, un profesor por siempre, como él mismo lo afirma en "Darío Ruiz Gómez, para buscar la ciudad", cuando le pregunto por la importancia de la disciplina en el escritor responde en coro con uno de sus autores favoritos:

Pavese dice que lo más importante en un escritor es el rigor de la disciplina que conduce al oficio de la escritura "así como se ama la vida por la vida". Y el rigor diario le permite a un escritor encontrar aquello que no buscaba en las palabras, esos reinos invisibles de la escritura que nos llevan a reconocer un magisterio y a sabernos contemporáneos de los grandes maestros "al buscar lo que ellos buscaban" (Vergara, 2021, p. 192). 
Y cerramos con Pablo Montoya Campuzano, cuya obra ya prolífica también da cuenta de su dedicación; ante nuestra consulta sobre la trascendencia de la disciplina en la escritura literaria, él afirma:

Sin disciplina en la escritura no es posible la construcción de una obra literaria. Cuando se tiene en cuenta la consecución de un proyecto, sea un libro o una obra misma, el sentido de un trabajo de todos los días es indispensable. En el caso mío es más ostensible si tenemos en cuenta, por ejemplo, el soporte de investigación qué hay detrás de mis novelas. Un libro puede nacer de una obsesión, de una imagen, de un dolor o de un trauma emocional. Situaciones de índole personal o incluso colectiva pueden generar el primer impulso de la escritura de un libro literario, pero luego viene esa labor que exige una organización del tiempo y una constante actividad para que el libro llegue al buen puerto que significa su finalización y su respectiva publicación. Sin disciplina, la escritura literaria sería tan solo un pasatiempo. La disciplina, a mi juicio, es lo que otorga seriedad y altura a nuestra labor de escritores (P. Montoya, 202I, comunicación personal con el autor).

Así pues, estimado lector, estimada lectora: si usted ha llegado a hacerse la pregunta que Jean Stein le hiciera a William Faulkner en 1956 respecto a si existe alguna fórmula para convertirse en un buen novelista, todos los testimonios presentados aquí apuntan a la misma respuesta: no existen fórmulas mágicas. La clave está en leer e investigar mucho, y aplicarse día a día, con una voluntad de hierro, al arte de la escritura. Sin esta disciplina, el talento no pasará de ser un diamante en bruto, porque hacer literatura exige convertirse en un verdadero tallador de diamantes, como lo muestran las obras de los escritores que hemos visitado en estas páginas.

\section{El número 50}

En esta edición presentamos en primer lugar a una mujer adelantada para su época; en "Mi infancia explica mi vida': Soledad Acosta de Samper y la narración de la niñez", Camila Gómez Zuleta indaga por las representaciones de los niños en algunos relatos de la escritora, incluyendo una evocación autobiográfica, para mostrar cómo ella contribuye a la creación de la categoría de infancia en Colombia. Y luego pasamos a la "Existencia y presencia de Maín Ximénez en la vida y obra Porfirio Barba Jacob”, donde Esnedy Aidé Zuluaga nos muestra la trascendencia que adquiere este seudónimo tempranero del poeta de Santa Rosa de Osos.

Y de la poesía pasamos a "Lectores y lectoras en la prensa comunista colombiana: redes epistolares en el periódico Tierra”, en el que Diana Paola Guzmán rastrea a unos lectores militantes y el modo como estos combinan la lectura y la escritura con la acción política. Y continuando con la lectura, Tania Camila Triana Cuevas y Julio 
Alberto Bejarano nos llevan a "El cuento colombiano en el Boletin Cultural y Bibliográfico: un recorrido por la crítica y las publicaciones en los años 60" para identificar las posturas de los críticos frente al cuento, y las tendencias formales y temáticas de los autores en aquellos años.

Por su parte Diego Alejandro Zuluaga nos permite asomarnos a una relación literaria e intelectual en "Rafael Humberto Moreno Durán y Rafael Gutiérrez Girardot: la correspondencia de un escritor y su crítico literario", donde nos muestra cómo el primero encuentra en el segundo la oportunidad de proyectar y legitimar su obra a través de una voz que ya había alcanzado notoriedad en el mundo académico. Y en una relación de contigüidad encontramos "Ficción vírica e inmunización social en los 'proyectos piloto' de Enrique Buenaventura y Enrique Lozano", donde Sandra María Ortega muestra cómo a través de estas obras dramatúrgicas se revela "una imagen metafórica que refleja la deshumanización de la sociedad colombiana y las presentes políticas sobre la vida".

Y finalizamos con unas obras literarias muy contemporáneas. Álvaro Antonio Bernal nos presenta "El artilugio de un relato policíaco intercalado en $\mathrm{El}$ sindrome de Ulises de Santiago Gamboa", novela en la que, advierte, un relato policíaco intercalado se convierte en un recurso narrativo que desemboca en una vertiente del género negro. Y cerramos con la lectura de Juan Alberto Conde, quien en "Cuerpos difusos. Una lectura queer de tres novelas de ciencia ficción colombiana” visita producciones muy recientes de Andrea Salgado, Diana Catalina Hernández y Luis Carlos Barragán, respectivamente, para fijar su atención en el modo como son representadas las sexualidades no binarias en dichas obras.

Este es, estimados lectores, el menú que les ofrece Estudios de Literatura Colombiana en esta edición 50, un número mágico que encuentra a la revista gozando de una gran vitalidad: hoy estamos en la privilegiada zona Q1 del ranking sJR, que nos encaminó también a la clasificación A1 de Publindex para este año 2022. Y si bien estos logros son importantes, no dejan de ser asuntos formales que al fin de cuentas resultan acartonados. Lo que realmente nos da esa vitalidad que nos llena de orgullo es la presencia de la revista entre ustedes y las colaboraciones que permanentemente nos llegan desde algunos de los escenarios más importantes para la literatura colombiana. Esto nos hace pensar que estamos cumpliendo con nuestro objetivo de contribuir y participar en el diálogo académico en torno a las literaturas de nuestro país. 
Así, una vez más tenemos que darles gracias a ustedes por su confianza, a los colaboradores, a los evaluadores, a los miembros del comité académico y del comité editorial y a la Facultad de Comunicaciones y Filología, nuestra casa, por el respaldo. Gracias también a Christian Benavidez Martínez, el editor asistente, por su compromiso con la revista.

A todos, gracias.

\section{Andrés Vergara Aguirre \\ Universidad de Antioquia \\ Director editor}

\section{Referencias bibliográficas}

Duras, M. (1994). Escribir. México: Tusquets Editores.

Díaz-Granados, J. L. (2017). Gabo: ejemplo de disciplina y perfección. Libros \& Letras. Revista Cultural de Colombia y América Latina. Recuperado de: https://www.librosyletras.com/2017/06/ gabo-ejemplo-de-disciplina-y-perfeccion.html [2021.12.12].

Escobar Velásquez, M. (2019). Diario de un escritor. Medellín: Editorial Universidad de Antioquia.

Faulkner, W. (1996). William Faulkner / Entrevistado por Jean Stein, 1956. En L. Chitarroni (Comp.). Confesiones de escritores: los reportajes de The Paris Review: Narradores 1 (pp. 37-54). Buenos Aires: El Ateneo.

Guillermoprieto, A. (2014). Entre la disciplina y la parranda. El País (19 de abril). Recuperado de https://elpais.com/cultura/2014/04/18/actualidad/1397819758_215292.html [17.12.2021].

Hemingway, E. (1996). Ernest Hemingway / Entrevistado por John Plimpton, 1958. En L. Chitarroni (Comp.). Confesiones de escritores: los reportajes de The Paris Review: Narradores 1 (pp. 83 105). Buenos Aires: El Ateneo.

Ruiz Gómez, D. (2021). Comunicación personal, 16 de diciembre.

Saiz, E. (2012). Los 47 finales de Adiós a las armas. El País (9 de julio). Recuperado de https://elpais. com/cultura/2012/07/09/actualidad/1341865293_880241.html [17.12.2021].

Vergara Aguirre, A. (2021). Darío Ruiz Gómez, para descubrir la ciudad. Estudios de Literatura Colombiana 50, pp. 181-193.

Yajure, A. (2014). “Gabito es hechura de su propia disciplina”. Albertoyajure (17 de abril). Recuperado de https://albertoyajure.wordpress.com/2014/04/17/gabito-es-hechura-de-su-propia-disciplina/ [17.12.2021]. 